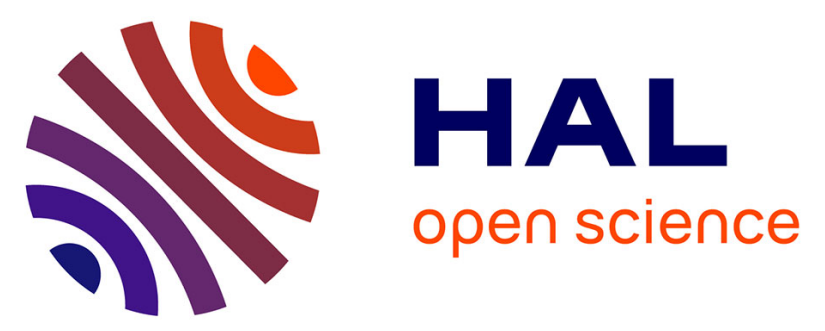

\title{
Dissipation measurements in steel sheets under cyclic loading by use of infrared microthermography
}

Martin Poncelet, Cédric Doudard, Sylvain Calloch, François Hild, Bastien

Weber

\section{- To cite this version:}

Martin Poncelet, Cédric Doudard, Sylvain Calloch, François Hild, Bastien Weber. Dissipation measurements in steel sheets under cyclic loading by use of infrared microthermography. Strain, 2010, 46, pp.101-116. 10.1111/j.1475-1305.2009.00622.x . hal-00356522

\section{HAL Id: hal-00356522 https://hal.science/hal-00356522}

Submitted on 27 Jan 2009

HAL is a multi-disciplinary open access archive for the deposit and dissemination of scientific research documents, whether they are published or not. The documents may come from teaching and research institutions in France or abroad, or from public or private research centers.
L'archive ouverte pluridisciplinaire $\mathbf{H A L}$, est destinée au dépôt et à la diffusion de documents scientifiques de niveau recherche, publiés ou non, émanant des établissements d'enseignement et de recherche français ou étrangers, des laboratoires publics ou privés. 


\title{
DISSIPATION MEASUREMENTS IN STEEL SHEETS UNDER CYCLIC LOADING BY USE OF INFRARED MICROTHERMOGRAPHY
}

\section{Running head title: THERMOGRAPHIC ANALYSIS OF DISSIPATION IN STEELS}

\author{
M. Poncelet,${ }^{(*)}$ C. Doudard, ${ }^{(* *)}$ S. Calloch, ${ }^{(*)}$ F. Hild, ${ }^{(*)}$ B. Weber ${ }^{(* \star)}$ \\ ${ }^{*}$ ) LMT-Cachan (ENS Cachan/CNRS/Université Paris 6/PRES UniverSud Paris) \\ 61 avenue du Président Wilson, F-94235 Cachan Cedex, France \\ (**) LBMS EA 4325 (E.N.S.I.E.T.A./Université de Brest/E.N.I.B.) 2 rue François Verny, 29806 Brest Cedex, \\ France \\ (***) ArcelorMittal Maizières ResearchSA, Voie Romaine BP 30320, 57283 Maizières-lès-Metz, France \\ Corresponding author: poncelet@Imt.ens-cachan.fr, fax +331474022 40
}

\begin{abstract}
Heterogeneous dissipation in steel sheets due to cyclic loading is difficult to measure, especially in the transverse direction because of the high conductivity and low thickness of the sheets. The goal of this article is thus to develop an experimental protocol allowing for the dissipation field determination from infra-red thermography. The protocol is based on a specific differential measurement and an asynchronous acquisition. It reduces measurement artefacts due to coating, rigid body motion, convection, and optical deleterious effects. It is eventually applied to different specimens.
\end{abstract}

KEY WORDS: IR thermography, thermoelasticity, dissipation, fatigue properties, surface effect, coating

\begin{tabular}{|c|c|c|c|}
\hline \multicolumn{4}{|c|}{ Nomenclature } \\
\hline$\Sigma$ & tensile stress & $\phi$ & radiation \\
\hline$\Sigma_{\max }$ & maximum tensile stress over a cycle & $\theta$ & temperature \\
\hline & loading ratio & $\theta_{m}$ & mean temperature over the specimen \\
\hline & loading frequency & $\begin{array}{l}\theta_{i n i} \\
T_{0}\end{array}$ & $\begin{array}{l}\text { temperature at initial state } \\
\text { absolute reference temperature }\end{array}$ \\
\hline $\begin{array}{l}\sigma_{\mathrm{y}} \\
\sigma_{\mathrm{UTS}}\end{array}$ & $\begin{array}{l}\text { yield stress } \\
\text { ultimate tensile stress }\end{array}$ & $\theta_{\text {ref }}$ & $\begin{array}{l}\text { reference temperature for sequence } \\
\text { selection }\end{array}$ \\
\hline & Young’s modulus & & \\
\hline$v$ & Poisson’s ratio & $\begin{array}{l}T_{C N} \\
\theta_{C N}\end{array}$ & $\begin{array}{l}\text { temperature of the black body } \\
\text { corresponding measured temperature }\end{array}$ \\
\hline $\begin{array}{l}\lambda \\
\alpha\end{array}$ & $\begin{array}{l}\text { thermal conductivity } \\
\text { thermal expansion coefficient }\end{array}$ & & on the specimen \\
\hline & $\begin{array}{l}\text { specific heat capacity } \\
\text { mass density }\end{array}$ & $\theta_{t h}$ & $\begin{array}{l}\text { variation of temperature due to } \\
\text { thermoelasticity }\end{array}$ \\
\hline & heat transfer coefficient & $\theta_{0}$ & temperature variation $\left(\theta_{t h}\right)$ amplitude \\
\hline & thermal characteristic time & $\begin{array}{l}\Delta_{t h} \\
D\end{array}$ & $\begin{array}{l}\text { thermoelastic dissipation } \\
\text { thermoelastic dissipation amplitude }\end{array}$ \\
\hline $\begin{array}{l}\lambda_{\text {paste }} \\
\lambda_{c}\end{array}$ & $\begin{array}{l}\text { thermal conductivity of paste } \\
\text { thermal conductivity of coating }\end{array}$ & $A$ & $\begin{array}{l}\text { temperature amplitude }\left(\theta_{0}\right) \\
\text { attenuation }\end{array}$ \\
\hline & $\begin{array}{l}\text { specific heat capacity of coating } \\
\text { mass density of coating } \\
\text { thickness of coating }\end{array}$ & $\xi$ & temperature variation $\left(\theta_{t h}\right)$ phase lag \\
\hline
\end{tabular}




\section{Introduction}

Despite numerous industrial needs, the understanding, modelling and characterisation of high cycle fatigue are still insufficient. The major reason for this fact is the cost (in time and money) of traditional fatigue experiments, i.e., more than a week of uninterrupted tests and several tens of specimens to get a Wöhler diagram. Alternative methods have thus been proposed to compensate for this difficulty.

A promising one, hereafter called "self-heating test," is based on the measurement of the specimen temperature changes during cyclic loadings [1-5]. For example, one uses two thermocouples to measure the differential temperature between the gauge zone of the specimen and the grips of the testing machine. The cyclic loading consists of a constant, stepwise amplitude that is increased once the temperature is stabilised. The steady-state temperature is plotted as a function of the loading amplitude as shown in Fig. 1 (a). For some materials (e.g., steels), a first part of the curve shows virtually no change in temperature, whereas in the second part the temperature increases significantly with the stress amplitude. A correlation between the mean fatigue limit and the stress level leading to the temperature increase has been empirically proposed [6-9]. Currently, models [10,11] based on microplasticity are developed to describe this result. To take into account the progressive onset of microplasticity a probabilistic approach is proposed [11], enabling one to relate the fatigue scatter to temperature measurements. Recently, this method was extended to multiaxial and non-proportional cyclic loadings [12,13].

Despite the importance of surface effects on endurance properties, this type of investigation has not yet been performed on specimens with surface effects. Therefore, the following study focuses on a high-strength boron steel sheet $(2 \mathrm{~mm}$-thick $22 \mathrm{MnB} 5$ produced by ArcelorMittal, $\sigma_{\mathrm{y}} \approx 1000 \mathrm{MPa}, \sigma_{\mathrm{UTS}} \approx 1500 \mathrm{MPa}$ ) whose surface is highly affected by the fabrication process. During hot-drawing, a surface layer of about $50 \mu \mathrm{m}$ is decarburised, leading to a low endurance limit (275 MPa under alternating tension at $2 \times 10^{6}$ cycles). The consequence of this partial decarburisation of the surface is well known [14] and is studied herein as a classic example. A steel sheet with the same chemical composition and thickness, but protected by an AlSi coating preventing decarburisation, is also studied (endurance limit under alternating tension at $2 \times 10^{6}$ cycles equal to $450 \mathrm{MPa}$ ) for comparison purposes. Specimens of coated and uncoated steel are prismatic with a rectangular cross section of $2 \times 8 \mathrm{~mm}$ (Fig. 1 (b)). The $8 \times 120 \mathrm{~mm}$ faces are not machined and thus have their original properties. The cut faces $(2 \times 120 \mathrm{~mm})$ are mechanically polished $(800$ sandpaper $)$ after waterjet cutting.

Figure 1 (a) shows self-heating curves of both specimens. Major differences between the two macroscopic measurements are observed. The partially-decarburised (non-protected) steel sheet temperature is higher, corresponding to a lower endurance limit when compared with the non-decarburised material. 


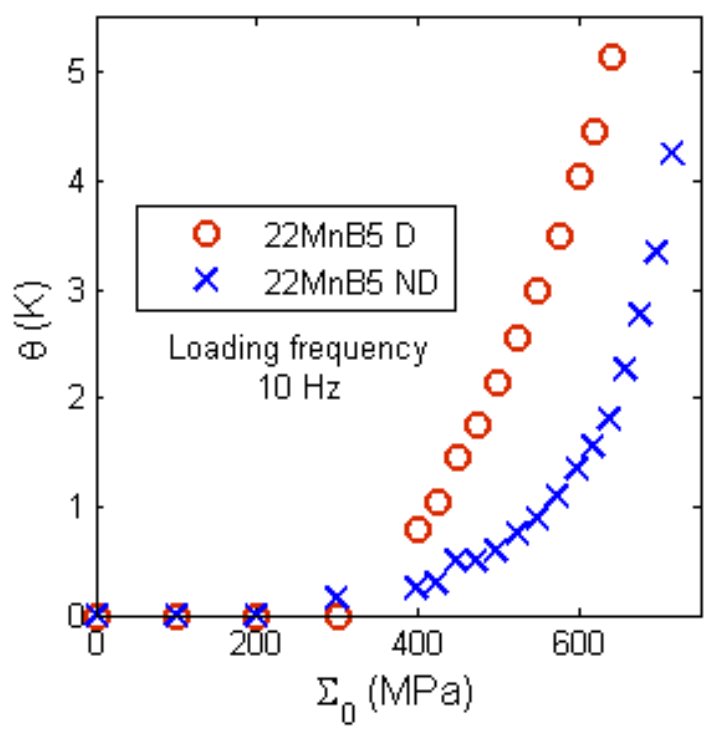

(a)

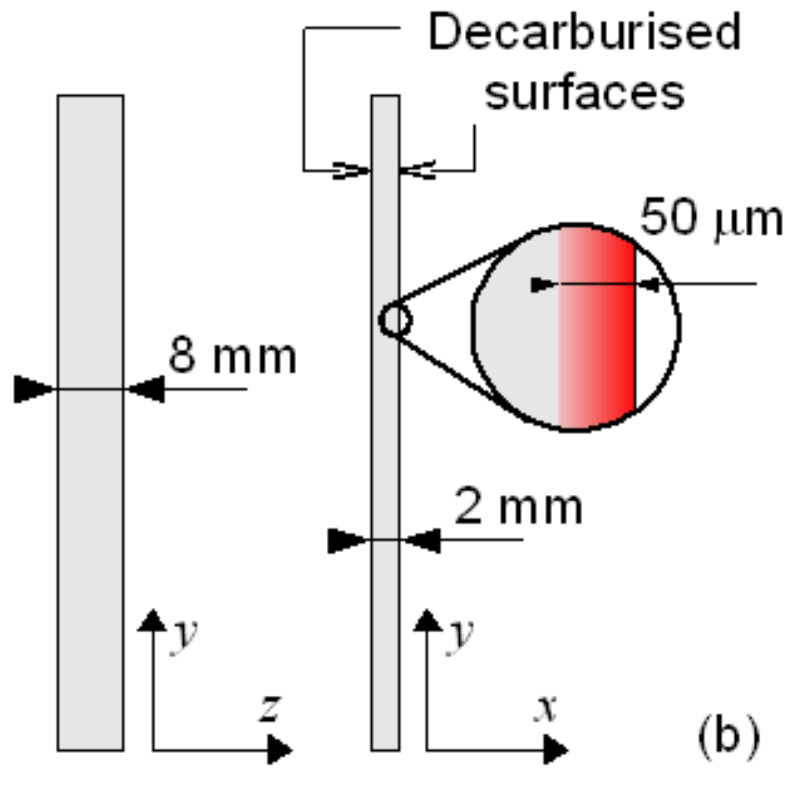

Figure 1: (a): Mean steady-state temperature $\theta$ of partially-decarburised (D) and nondecarburised (ND) 22MnB5 specimens under cyclic loading for different stress amplitudes $\Sigma_{0}$. (b): Design of the $22 \mathrm{MnB} 5$ steel specimen.

However, the direct prediction of endurance limits with an average analysis is only possible in uniform cases [2]. For heterogeneous specimens, one has to solve the heat equation to find the heat source distribution, and thus information on the temperature field is needed. As shown later, the temperature measurement "within" the steel sheet (i.e. in the transverse direction) is very difficult because of the thickness and conductivity of steel sheets. Consequently the computation of the heat equation, very sensitive to noise, is awkward, and the following identification of fatigue properties is almost impossible. The goal of this paper is thus to develop an experimental protocol allowing for particular temperature field measurements under cyclic loading that can be related to the dissipation field, provided that it reduces measurement artefacts to their minima, and to apply it to different specimens (the heat conduction equation computation and the fatigue properties identification are not in the scope of the present paper).

We will describe step-by-step the development of this experimental protocol [15]:

- First, the principle of the temperature measurement is presented.

o The experimental equipment, namely the IR camera and the testing machine, is described.

o The common differential measurement being insufficient herein, the main idea is to perform the "most differential measurement" to reduce noise and artefacts.

o An asynchronous acquisition is used to prevent spurious effects of potentially heterogeneous thermoelasticity.

- Second, the artefacts prevention is explained. It consists of the correction for:

o Rigid body motions, which blur the temperature measurement.

o High emissivity coating effects, due to the heat conduction through it during cyclic loadings.

o Convection, whose non-constant influence will be reported to be tremendous. 
o Optical artefacts, which may appear when a contrasted scene it measured.

Last, the protocol is applied to an artificially heterogeneous specimen and to partiallydecarburised steel sheets. Results are then discussed.

\section{Principle of measurement}

The geometrical configuration of the experimental set-up is described using the following set of axes (Fig. 1 (b)), namely, $x$ is the transverse direction of the steel sheet, $y$ the loading axis oriented upward and $z$ the optical axis oriented toward the camera. The $(x, y)$ frame is thus the measurement plane and the $(y, z)$ plane is that of the steel sheets.

From a theoretical point of view, the temperature measured on the $z=0$ plane is not representative of that in the bulk (i.e. in $z<0$ planes). However, the heat transfer through the surface $z=0$ (convection and radiation) being very low compared with the inner one (conduction), and the dissipation heterogeneity being independent of $\mathrm{z}$ coordinate, one assumes that the temperature heterogeneity is independent of $z$ coordinate. The temperature field measured on the surface $z=0$ is thus assumed to be representative of the $z$-average temperature field in the bulk $(z<0)$.

Two major issues appear when attempting to carry out a temperature measurement on such configurations of thin metal sheet:

- Considering only the gradient due to convection (heat transfer coefficient $h \approx 2 \mathrm{~W} \cdot \mathrm{m}^{-2} \cdot \mathrm{K}^{-1}$ ) in the case of a uniform 2-mm-thick steel sheet (conductivity $\lambda \approx 45 \mathrm{~W} . \mathrm{m}^{-1} \mathrm{~K}^{-1}$ ), Biot's number is about $10^{-4}$, i.e. the temperature variations are very small $\left(<10^{-2} \mathrm{~K}\right)$. As a consequence, the principle of the measurement must reduce artefacts and noises to their minimum level in order to get any meaningful information. Moreover the temperature variation due to thermoelastic coupling being far more important than the sought ones, one has to ensure that the thermoelastic phenomenon has strictly no influence on the final measured temperature.

- Second, because of the fixed and short working distance of the microscopic lens described hereafter $(40 \mathrm{~mm})$, a standard vacuum chamber cannot be used to prevent convection. The influences of the steady-convection and of the heterogeneity of dissipation are equivalent in terms of temperature magnitude. Moreover, the flow of heat is difficult to estimate accurately in the case of natural convection at room temperature (one usually assumes that the coefficient of linear convection $h$ varies between $0.2 \mathrm{~W} \cdot \mathrm{m}^{-2} \cdot \mathrm{K}^{-1}$ and $\left.2 \mathrm{~W} \cdot \mathrm{m}^{-2} \cdot \mathrm{K}^{-1}[16]\right)$. In addition, $h$ may change with the experimental conditions. It is therefore difficult to take convection into account $a$ posteriori.

One thus needs experimental equipment with adequate thermal and spatial resolutions, and uncertainties. Moreover one needs a specific strategy to measure the temperature gradient due to the (intrinsic) heterogeneity of the microplastic behaviour and not extrinsic conditions or thermoelastic coupling.

\section{Experimental equipment}

The two fundamental elements of the set-up are the testing machine and the infra-red camera. Cyclic loading is applied with an MTS servohydraulic testing machine equipped with mechanical grips. Static flexure of the specimen is prevented by accurate alignment of the grips. There was no dynamic flexure in the specimen caused by the eigenmodes of the test machine. This was confirmed using a high speed camera (Photron ultima APX-RS, $1024 \times 1024$ pixels, $3 \mathrm{kHz}$ in full frame mode) and a Digital Image Correlation (DIC) software to obtain strain fields under the usual loading conditions $(500 \mathrm{MPa}$ at $30 \mathrm{~Hz})$. 
The camera is an FPA MWIR camera (Cedip Jade III) with an InSb $320 \times 240$ element detector, cooled at $70 \mathrm{~K}$ with a Stirling device. The maximum number of frame per second in full frame mode is about 150. The Noise Equivalent Thermal Difference (NETD) given by the manufacturer is around $20 \mathrm{mK}$ at $20{ }^{\circ} \mathrm{C}$. Due to the size of the specimen, the camera is equipped with a standard microscopic lens (Cedip G1). Its magnification is 1 so that a pixel is equivalent to a $30 \times 30 \mu \mathrm{m}$ zone on the specimen surface. An adequate integration time is searched for, enlarging the thermal resolution to it maximum without saturation of any of the pixels. This enables us to find an integration time equal to $1640 \mu \mathrm{s}$, enhancing the thermal resolution on the $20^{\circ} \mathrm{C}-40^{\circ} \mathrm{C}$ range by a factor 2 , and lowering the measurement uncertainty by a factor 2.5 in comparison with the initially proposed integration time (700 $\mu \mathrm{s})$.

The calibration is an individual (i.e., pixel-wise) polynomial fit of the response of the detector elements [17] instead of the embedded linear non uniformity correction [18]. The reference instrument is a planar black body (DCN1000 by $\mathrm{HGH}$, active surface: $100 \times 100 \mathrm{~mm}$, spatial non-uniformity: $\pm 0.01 \mathrm{~K}$ at room temperature $\pm 5 \mathrm{~K}$, stability: $\pm 0.002 \mathrm{~K})$. The identification of 6-degree polynomials is performed on a series of images corresponding to 21 regularly-spaced temperatures of the black body over the range $20^{\circ} \mathrm{C}$ $40^{\circ} \mathrm{C}$. Each of them is the result of time averages of a 600 -frame movie to decrease temporal noise.

Under these conditions, the space-averaged RMS value of temporal noise (19 $\mathrm{mK})$ is equivalent to the given NETD, i.e. greater than the sought temperature difference. Since one searches only temperature variations in the transverse direction of steel sheets, and since the sheets are uniform in the other directions, it is possible to perform a space-average of the images in $y$ direction (i.e. loading direction). The measurement error is a white Gaussian noise, consequently this average over 320 pixels of a line lowers the uncertainty level to $19 / \sqrt{320} \approx 1.1 \mathrm{mK}$, which is considered sufficient.

\section{Need for the "most differential measurement"}

Using the experimental set-up without any of the proposed correction described hereafter (i.e., simply the IR camera, the testing machine and a high emissivity coating), one measures during the test the temperature field of the specimen $\theta(x, y, t)$. The simplest protocol is a direct (non-differential) measurement of the temperature of the steel sheet under cyclic loading followed by a time average to reduce the measurement uncertainty. Figure 2 (a) shows a typical temperature field $\theta(x, y)$ where the specimen under cyclic loading appears hotter than the background. Despite the coating covering the specimen, the Narcissus effect is mainly responsible for the measured heterogeneity. Its influence on $x$ temperature variations seems low, as shown in Fig. 2 (a). However, plotting the $y$-average temperature in Fig. 2 (c), one clearly sees that $\theta(x)$ is non symmetrical, with $\max _{x} \theta-\min _{x} \theta \geq 0.25 \mathrm{~K}$, i.e. far more than the sought heterogeneity $\left(<10^{-2} \mathrm{~K}\right)$.

The usual way to get rid of Narcissus artefacts is to perform a differential measurement between the state under cyclic loading $\theta(x, y)$ and the initial state $\theta_{\text {ini }}(x, y)$. In that case, the differential temperature field $\theta(x, y)-\theta_{\text {ini }}(x, y)$ is far more uniform (note the change of temperature scales in Fig. 2 (b)). The main gradient is oriented along the $y$ direction, and corresponds to the heat flux coming from the actuator. Looking at the $y$ average in Fig. $2(\mathrm{~d})$, the temperature variation $\theta(x)-\theta_{i n i}(x)$ is more symmetrical and $\max _{x}\left(\theta-\theta_{\text {ini }}\right)-\min _{x}\left(\theta-\theta_{\text {ini }}\right) \leq 0.05 \mathrm{~K}$. However, this temperature field clearly does not correspond to the sought one, where $\max _{x}\left(\theta-\theta_{\text {ini }}\right)-\min _{x}\left(\theta-\theta_{\text {ini }}\right) \leq 0.01 \mathrm{~K}$. 


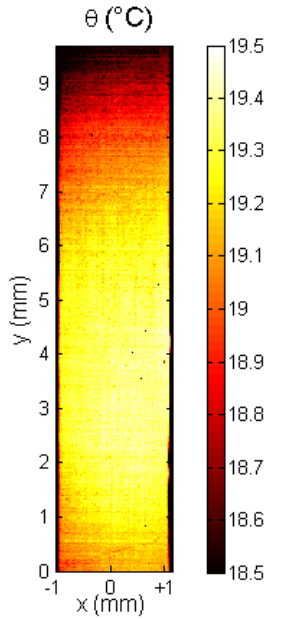

(a)

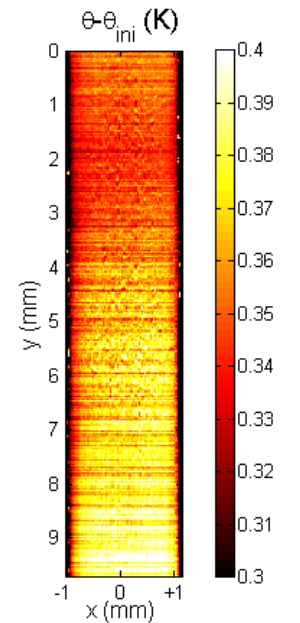

(b)

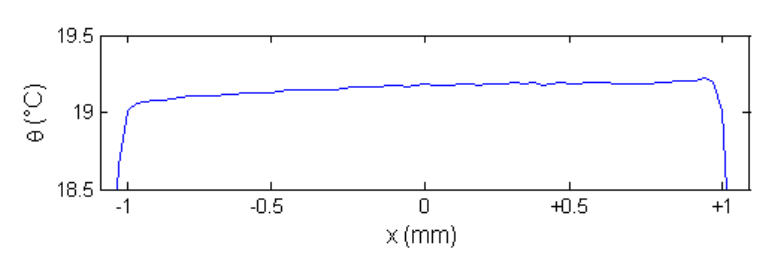

(c)

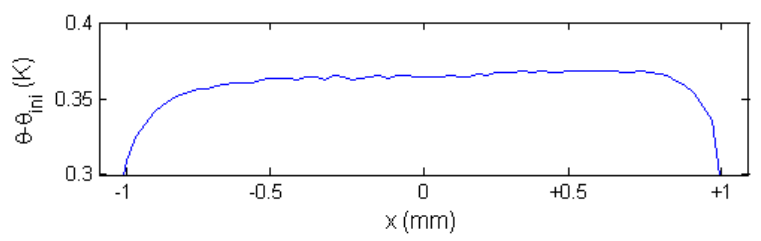

(d)

Figure 2: (a) Direct measurement of the temperature of the specimen after about $4 \mathrm{~s}$ of loading $\left(R=-1, \Sigma_{\max }=500 \mathrm{MPa}, 30 \mathrm{~Hz}\right)$; (b) Differential temperature of the specimen between about $4 \mathrm{~s}$ of loading and initial state; (c) $y$-average of the direct measurement of the temperature; (d) $y$-average of the differential measurement of the temperature.

Except for the appearance of heat source heterogeneity, the major difference between the initial and loading state is the mean temperature of the specimen (around $0.35 \mathrm{~K}$ in the case of Fig. 2) due to the self-heating effect itself. All these phenomena, which are related to the temperature of the specimen (e.g. convection, radiation, but also optical artefacts), are not deleted by the differential measurement. Consequently, a strategy is proposed where the differential measurement is performed between two states of the specimen at the same mean temperature (later called "most differential measurement"), so that the difference of the latter phenomena will be negligible to the first order.

The strategy followed is shown in Fig. 3. During a first stage, the specimen is loaded cyclically and its average temperature increases slowly (the steady-state temperature is reached after several tens of seconds, depending on the geometry of the specimen, and on material and thermal conditions). On the contrary, numerical simulations show that the temperature heterogeneity appears very quickly (less than several hundredths of one second) and then evolves slowly as the average temperature increases the role of convection, namely, the initial concave profile (temperature of the surfaces greater than that in the bulk due to a higher level in heat sources) turns into a convex one (heat losses by convection and radiation "bypass" heat sources on surfaces). This phenomenon is experimentally checked by subtracting two temperature fields taken under loading for different mean temperatures (i.e. the second one later than the first one, so that the second one is hotter than the first one) and the observed difference is not equal to zero. During the second stage, the specimen is not loaded and its average temperature returns slowly to the initial one due to heat losses. As in stage I, the temperature heterogeneity changes quickly after the loading stops, then the same slow phenomenon occurs as the average temperature tends to the room temperature. 

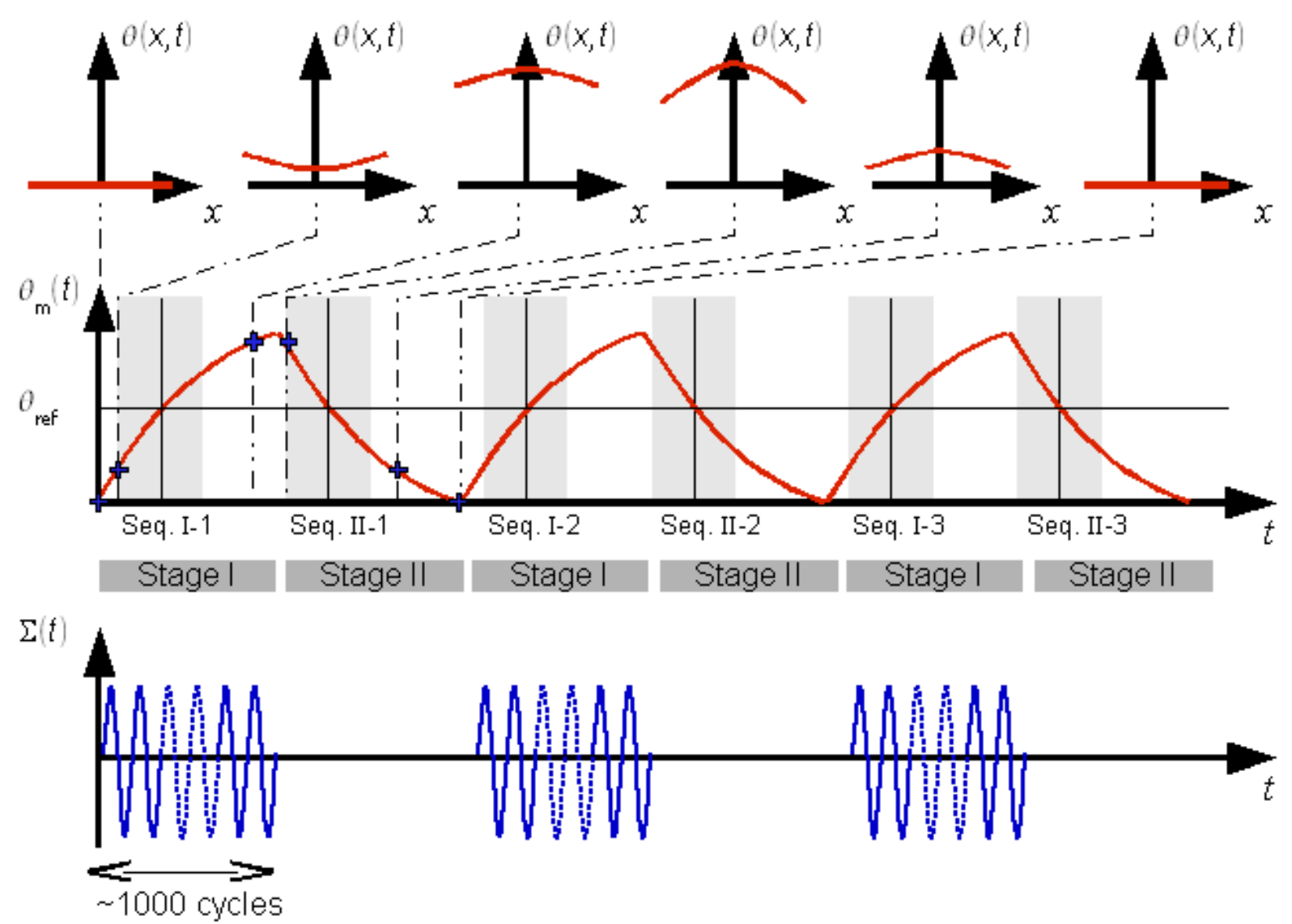

Figure 3: Strategy of measurement: Tensile stress $\Sigma(t)$ (bottom). Change of mean temperature $\theta_{m}$ (middle) with time $t$ during stages I and II. Corresponding heterogeneity $\theta$

(top) during the first cycle of stage I and II. The heterogeneity changes slowly during the increase and decrease of the mean temperature. Repeated short series of stages I and II reduce the mean temperature variations.

Temperature acquisition is performed during stages I (heating) and II (cooling). One sequence is selected during each stage so that the time-and-space average temperature of the specimen is the same for each sequence and equal to a reference temperature $\theta_{\text {ref. }}$. Unless convection conditions change between stages I and II, the heat losses by linearised convection and radiation are thus nearly identical during the two sequences and the effect of convection is "removed" by subtraction of sequences. More precisely, heat losses are not strictly speaking identical, but the relative difference between the heat losses during stage I and II is equivalent to the ratio "heterogeneity magnitude due to the heat sources" / "mean temperature of the specimen", in other words, negligible. More generally, every artefact linearly depending on the temperature of the specimen is virtually deleted by following the same strategy. Last, this "most differential measurement" method ensures for results independent of the choice of the reference $\theta_{r e f}$. It is important to note that the result of this protocol does not represent the absolute temperature field of the specimen, but only the differential temperature between stages I and II.

Noise (e.g.., measurement uncertainty and turbulent convection) is mainly reduced by time averages. For long time averages (e.g. several tens of seconds) one single long stage I followed by a long stage II could be performed. However, this protocol leads to higher mean temperatures at the end of stage I, which may disturb the initial heat transfer conditions. It is 
preferred to conduct several short series of stages I and II without reaching steady-state conditions. For example, 10 series of stages I and II of about $8 \mathrm{~s}$ each are performed for the following application to $22 \mathrm{MnB} 5$ samples. This second method allows one to accumulate a higher number of frames in a fragmented way, and thus without major variations of the mean temperature. However uncertainty and artefacts reductions are also achieved by appropriate acquisition mode, and data-processing as shown in the next section.

\section{Asynchronous acquisition}

Because only steady-state temperature fields due to microplastic dissipation are searched for (and not the thermoelastic component), one may wonder whether to use lock-in acquisition. It has been shown [19] that lock-in thermography is a very interesting method when macroscopic temperature fields are sought. In the present case, lock-in thermography may create artefacts due to a combination of heterogeneous thermoelastic dissipation and conduction. However not proven, such an heterogeneity may occur in a partially decarburised steel sheet because of the heterogeneity of the different material properties involved in the thermoelastic coupling (coefficient of thermal expansion $\alpha$, Young's modulus $E$, but also mass density $\rho$, and specific heat capacity $C$ ). Moreover, the strain being uniform in tension, a heterogeneity of Young's modulus leads to a heterogeneity of stress. We consequently study the influence of thermoelastic dissipation heterogeneity on the measured temperature field in the present section.

The general framework used to describe thermoelastity is given in Ref. [20]. If temperature variations are small $\left(\theta / T_{0} \ll 1\right.$, where $\theta$ is the temperature variation, and $T_{0}$ the absolute reference temperature), the thermoelastic dissipation reads

$\Delta_{t h}=-T_{0} \alpha\left(\operatorname{trace}(\dot{\Sigma})+\alpha \dot{\theta} \frac{3 E}{1-2 v}\right)$

where $v$ is Poisson's ratio, and $\Sigma$ the stress tensor. Dotted variables correspond to their first derivative with respect to time. For steels and small variations of $\theta, 3 E \alpha \dot{\theta} /(1-2 v)$ is negligible, so that the thermoelastic dissipation in tension reduces to

$\Delta_{t h}=-T_{0} \alpha \dot{\Sigma}$

Consequently, heterogeneous thermoelastic dissipation under sinusoidal loading at frequency $\omega_{S}$ reads

$\Delta_{t h}(x, t)=D(x) \sin \left(\omega_{s} t\right)$

where $D(x)$ takes into account the heterogeneity of material properties. It is now possible to introduce this dissipation in the heat equation

$\dot{\theta}_{t h}(x, t)-\frac{1}{\tau_{e q}} \theta_{t h}(x, t)-\frac{\lambda}{\rho C} \theta_{t h}(x, t)_{, x x}=\frac{D(x)}{\rho C} \sin \left(\omega_{s} t\right)$

where $\tau_{e q}$ is a characteristic time depending on the material and geometry of the specimen and its boundary conditions [16]. $; x x$ is the second derivative with respect to $x$-direction.

As a first approximation, convection and radiation are neglected on both sides of the specimen $\theta( \pm L / 2, t)_{, x}=0, L$ being the thickness of the specimen. This assumption is all the more relevant when temperature variations are fast (about $0.016 \mathrm{~s}$ for half a period at $30 \mathrm{~Hz}$ ). The solution to this problem reads

$\theta_{t h}(x, t)=\sum_{k=0}^{\infty}\left(\frac{D(x)}{\rho C}, C_{k}(x)\right) \frac{\omega_{s}}{\omega_{s}^{2}+\Omega_{s}^{2}}\left[e^{-\Omega_{k} t}-\left(\cos \left(\omega_{s} t\right)-\frac{\Omega_{k}}{\omega_{s}} \sin \left(\omega_{s} t\right)\right)\right] \times C_{k}(x)$ 
where $C_{k}(x)=\frac{\cos \left(\omega_{k} x\right)}{\int_{-L / 2}^{L / 2} \cos ^{2}\left(\omega_{k} x\right) d x}$ with $\omega_{k}=\frac{2 k \pi}{L}$ and $\Omega_{k}=\frac{1}{\tau_{e q}}+\omega_{k}^{2} \frac{\lambda}{\rho C}$. The term $e^{-\Omega_{k} t}$ corresponds to the transient state, whereas $\cos \left(\omega_{s} t\right)$ and $\sin \left(\omega_{s} t\right)$ are respectively the inertia and forced state conduction contributions. As shown in Table 1, the value of the conduction contribution is important even for the first harmonic, inducing a heterogeneous phase lag so that lock-in may create artefacts.

Table 1: Conductivity contribution $\frac{\Omega_{k}}{\omega_{s}}$ and attenuation $\frac{\omega_{s}}{\omega_{s}^{2}+\Omega_{s}^{2}}$ for different harmonics $k$. $\left(\tau_{e q}=40 \mathrm{~s}, L=2 \mathrm{~mm}, \lambda=50 \mathrm{~W} \cdot \mathrm{m}^{-1} \cdot \mathrm{K}^{-1}, \rho=7800 \mathrm{~kg} \cdot \mathrm{m}^{-3}\right.$ and $\left.C=500 \mathrm{~J}^{\mathrm{kg}} \mathrm{kg}^{-1}, \omega_{s}=10 \pi \mathrm{rad} . \mathrm{s}^{-1}\right)$

\begin{tabular}{|c|c|c|}
\hline$k$ & $\frac{\Omega_{k}}{\omega_{s}}$ & $\frac{\omega_{s}}{\omega_{s}^{2}+\Omega_{s}^{2}}\left(10^{-3}{\left.\mathrm{~s} \cdot \mathrm{rad}^{-1}\right)}^{-1}\right.$ \\
\hline 0 & 0.000792 & 5.77 \\
\hline 1 & 4.01 & 2.65 \\
\hline 2 & 16.0 & 0.877 \\
\hline 3 & 36.1 & 0.416 \\
\hline
\end{tabular}

To experimentally check this effect, a custom-made specimen is designed (Fig. 4), presenting a priori a heterogeneity of material properties depending only on the transverse direction $x$. It consists of a sandwich of 2 different steel sheets, a thinner version of the previous non-decarburised boron steel (thickness $=1.6 \mathrm{~mm}$ ) in the centre and an interstitial free steel (thickness $=2 \mathrm{~mm}$ ) on both sides. To ensure a single-piece-like thermal behaviour, a thin film of high conductivity thermal paste (around $10 \mu \mathrm{m}, \lambda_{\text {paste }}=25 \mathrm{~W} \cdot \mathrm{m}^{-1} \cdot \mathrm{K}^{-1}$ ) is put between the three sheets. Last, it is covered with a high emissivity coating described hereafter.

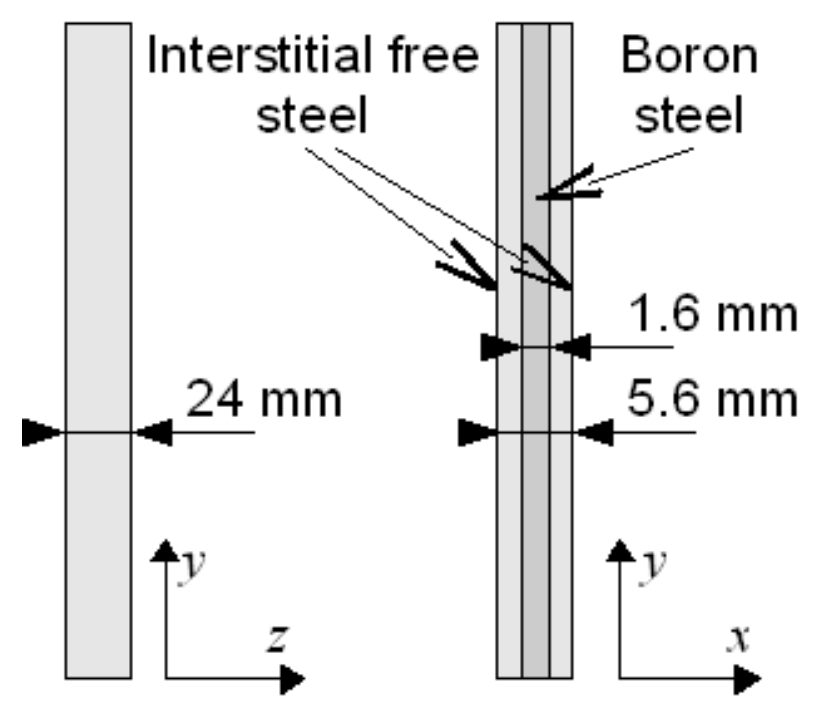


Figure 4: Design of the custom-made sandwich specimen.

In the present section, one is only interested in the variation of temperatures due to thermoelasticity (contrary to the remainder of this work). This "thermoelastic temperature" field of the specimen measured during a cyclic loading (stress amplitude $100 \mathrm{MPa}$ at $5 \mathrm{~Hz}$ ) is shown in Fig. 5. One observes both heterogeneity of amplitude and phase lag as predicted by Equation (5). In Fig. 5, the solution of the model is also presented. The chosen heterogeneity is such that $D$ is constant by step $([-0.8,+0.8] \mathrm{mm}$ for the central part, $[-2.8,-0.8[\mathrm{~mm}$ and ]$+0.8,+2.8] \mathrm{mm}$ for the outer parts). The chosen magnitude of the dissipation follows macroscopically the loading [Equation (2)]. However all material properties of each steel needed for the calculation of $D$ are not all well known, it is still possible to assume that the thermoelastic dissipation amplitude $D$ is globally $10 \%$ higher in the outer sheets than in the central one (e.g. because of difference of Young's modulus, non-linearity of thermoelasticity). Model results and measurements are in good agreement and confirm that triggering at zeroload is unable to "erase" thermoelasticity. Equation (5) proves that the thermoelastic temperature still vanishes over one cycle, hence averaging images taken at a high frequency during several loading cycles is a way of eliminating this contribution. The frequency of the camera and the testing machine are thus chosen coprime to enhance the sampling of a cycle.

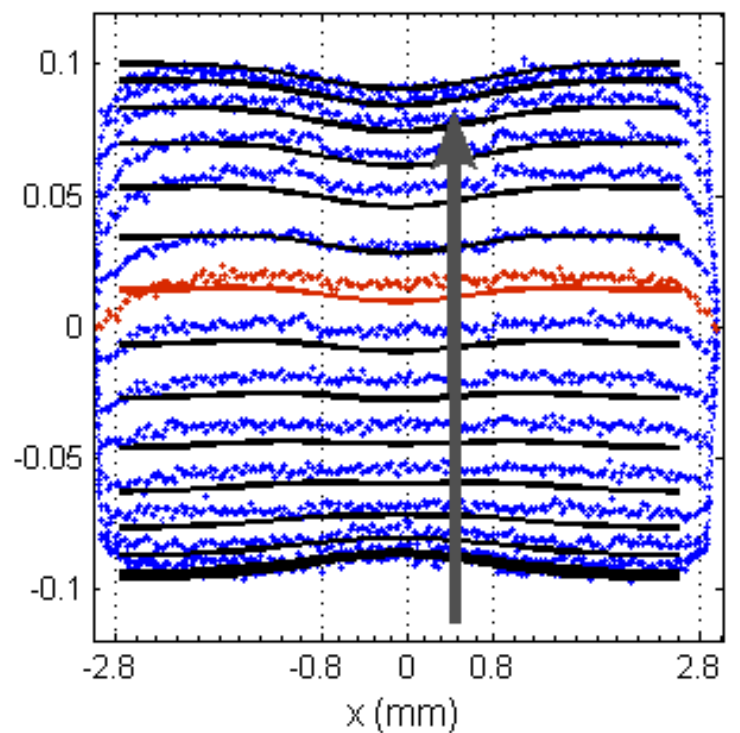

(a)

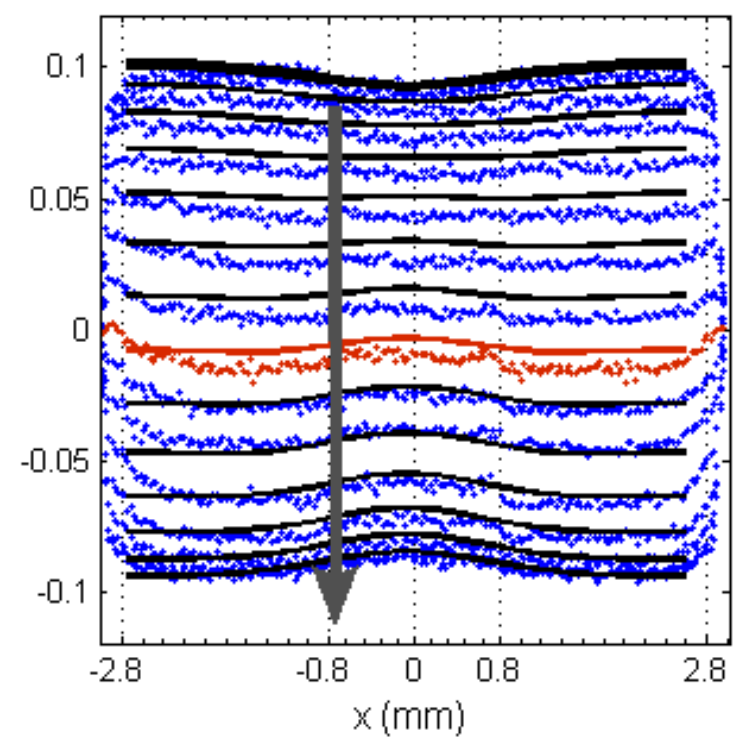

(b)

Figure 5: Temperature fields of the custom-made specimen under cyclic tensile loading (100 MPa, $5 \mathrm{~Hz}$ ). Only the cycle of the temperature field is plotted, corresponding to the "thermoelastic temperature". Dots are experimental data, and solid lines are the response of the model. The rise in temperature during half of a cycle is shown in (a) and the decrease during the other half in (b).

However, it can be noted that the current model does not explain the important heterogeneity near both edges of the specimen. This difference will be explained later on by accounting for the role of the coating.

The experimental equipment necessary to perform the measurement will be described in the next section. Every component of this set-up will be then justified by a study of different artefacts and uncertainties. 


\section{Artefacts prevention}

Despite the care exercised for data acquisition and further processing, one may encounter several artefacts. The first one, due to small displacements of the camera during the loading, will be corrected by Digital Image Correlation (DIC). The second one (due to coating during dynamic loading) is already prevented by asynchronous acquisition, yet it will be presented because of its delicate aspect and major importance. The third point is the characterisation and reduction of "convection noise". Last, different corrections of optical artefacts are briefly presented. These different corrections and preventions will need additional experimental equipments. This new set-up, shown in Fig. 6, is composed of:

- In its centre, the specimen.

- Along the $y$-axis, two grips and the actuator. A water-cooling ring is put between the lower grip and the actuator, and two thermocouples are fixed at the ends of the grips. It is thus possible to monitor and modify the temperature of the grips.

- Along the $z$-axis, the IR camera and its lens, focused on the edge of the steel sheet. A black body is placed about $120 \mathrm{~mm}$ behind the specimen to ensure a constant background radiation.

- On the side of the grips, a fan is fixed next to the upper grip, blowing air from behind and in front of the specimen. It homogenises the air inside the measurement area and improves time-averages applied a posteriori.

- Around the whole set-up (not shown in Fig. 6) a thick cotton fabric wraps the grips to prevent the thermal conditions inside the measurement area from being disturbed (convection and radiation).

The testing machine and the camera were previously described. All the other elements are described and justified in the following sections by an extensive study of the different sources of spurious effects, namely unwanted rigid body motions, high emissivity coating, turbulent convection and optical artefacts.

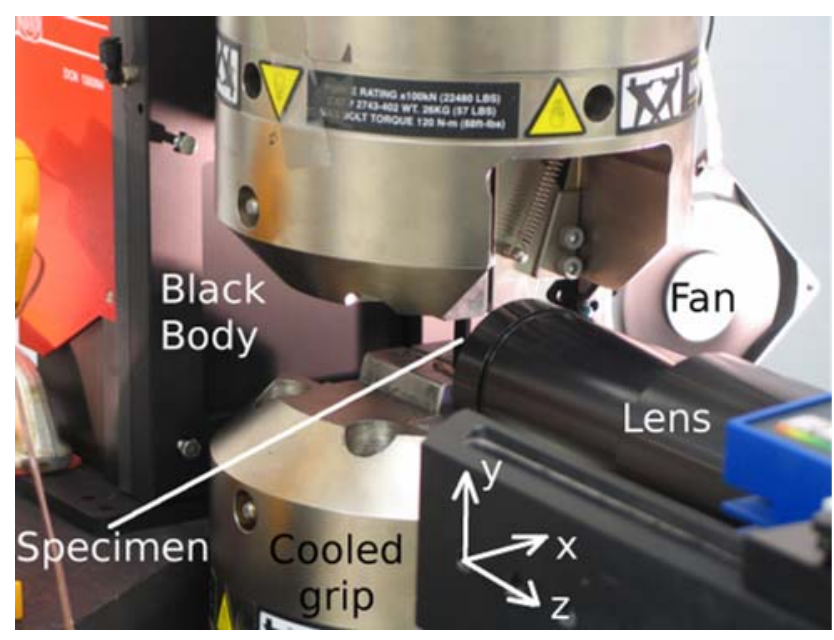

Figure 6: Complete experimental set-up with specimen in place, without the fabric.

\section{Rigid body motion correction}

Because of the frequency of loading, motions due to eigenmodes of the set-up are unavoidable. Even though no additional flexure or torsion of the specimen are induced by these motions, a rigid body motion of the specimen in the framing comes from the small displacements of the camera in front of the testing machine, despite a stiff and heavy tripod. These rigid body motions are reduced to a few pixels due to adequate clamping of the tripod 
to the testing machine. However it is necessary to correct for these motions to avoid artefacts due to subtraction.

The whole second sequence of frames being shot after loading, the specimen is perfectly still during this sequence. A time average is thus directly performed. The obtained image is then turned so that the axis of the specimen matches the $y$-axis of the camera (this correction is very small, less than $\tan ^{-1}(1 / 320) \sim 3 \times 10^{-3}$ rad., i.e. an error of 1 pixel along the $x$-axis over 320 pixels along the $y$-axis). This last image is then used as a reference for the rigid body correction of the first sequence. Every image of the first sequence is reset by a subpixel DIC algorithm [21] applied on the IR images. Because only rigid body motions are search for, one uses the high contrast between the background and the specimen to follow the displacement of the latter. This method enables to keep the uniformity of the high emissivity coating, contrary to use of "markers" with different emissivity [22,23]. Last, a time average is performed on the entire corrected first sequence. Subtraction of the two images (time average of the first and second sequences) is then operated.

The difference between corrected and non-corrected results for a $22 \mathrm{MnB} 5$ sample reaches several tens of $\mathrm{mK}$ as shown in Fig. 7. For the sake of comparison, the subtraction of corrected and raw fields of the second sequence is also presented. As expected, it shows very little difference since the specimen is motionless during the second sequence. This correction is hence necessary for the first sequence.

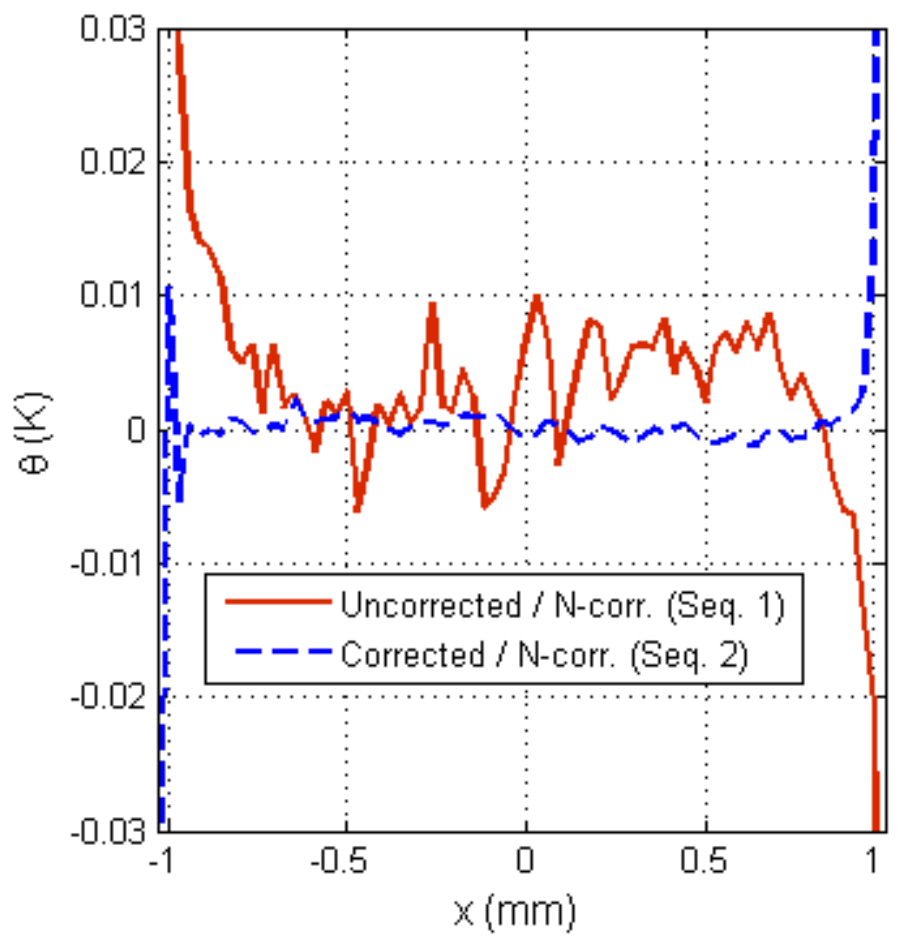

Figure 7: Influence of rigid body motion correction:

"temperature error" given by subtraction of corrected and raw sequences, extracted from stage I (Seq. 1) and stage II (Seq. 2).

\section{Role of coating under dynamic loading}

The choice of a high emissivity coating for any specimen is a crucial point in infra-red measurements. Several types were tested and one of them is a layer of carbon nanoparticules (typical diameter ranging from 10 to $400 \mathrm{~nm}$ ) with a thickness of about $50 \mu \mathrm{m}$. Its emissivity 
is reported in the literature [24,25] to be $\approx 96 \%$, and its uniformity is far better than conventional high emissivity paints at a microscopic scale.

To explain the temperature gradient near the edge of the specimen shown in Fig. 5 (phenomenon observed on every sample), the thickness of the coating must be taken into account. One observes in Fig. 8 that the thickness is not constant on the specimen surface, especially near the edges. This heterogeneity is due to the edge itself, creating vortices during the accumulation of nanoparticules. To model the thermal behaviour of the coating in a forced state, it is assumed that it is a uniform layer (conductivity $\lambda_{c}=0.1 \mathrm{~W} \cdot \mathrm{m}^{-1} . \mathrm{K}^{-1}$, mass density $\rho_{c}=250 \mathrm{~kg} \cdot \mathrm{m}^{-3}$ and specific heat $C_{c}=700 \mathrm{~J} \cdot \mathrm{kg}^{-1}$ ) of thickness $L_{c}$. It is assumed that the coupling between the specimen and the coating is weak, i.e. the temperature of their common interface $z=-L_{c}$ is prescribed by the thermoelastic behaviour of the specimen

$\theta\left(z=-L_{c}\right)=\theta_{0} \sin \left(\omega_{s} t\right)$

where $\theta_{0}$ is the amplitude of the temperature variation. The second boundary condition ( $z=0$, interface coating/air) is assumed to be with zero flux (no convection nor radiation)

$\theta(z=0)_{, z}=0$

The forced solution to this problem of pulsed mode is given by [26]

$\theta_{c}(z, t)=\theta_{0} A(z) \sin \left(\omega_{s} t+\xi(z)\right)$

with $A(z)=\left|\frac{\cosh k z(1+i)}{\cosh k L_{c}(1+i)}\right|$ and $\xi(z)=\arg \left[\frac{\cosh k z(1+i)}{\cosh k L_{c}(1+i)}\right]$

where $i$ is the imaginary unit. $A$ and $\xi$ are the attenuation of the temperature amplitude and the phase lag at a given position $z$ in the coating layer. It is worth noting that these two expressions are not linear functions of $z$, but depend also on the total thickness of the coating $L_{c}$.

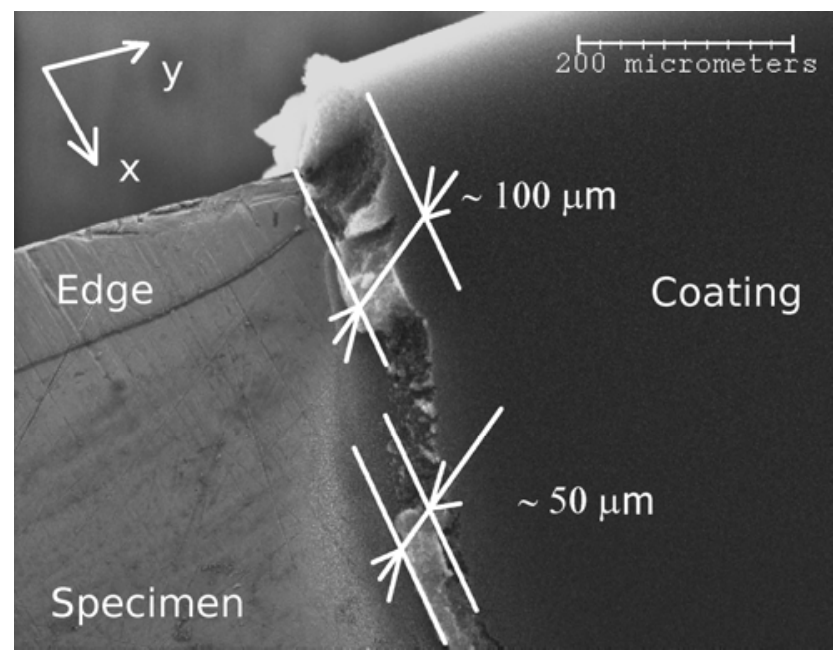

Figure 8: Thickness heterogeneity of the coating near a specimen edge.

By measuring the coating thickness (Fig. 8), one predicts the phase lag $\xi$ and attenuation $A$ of the temperature signal for a thick layer (near the edge, $\xi(0)=-0.300 \mathrm{rad}$, $A(0)=0.9702$ ) and a thin layer (in the centre, $\xi(0)=-0.076 \mathrm{rad}, A(0)=0.9980$ ). It is also possible to compare them to measurements carried out in exactly the same conditions. Phase lag differences are close ( $0.22 \mathrm{rad}$ for the model, $0.17 \mathrm{rad}$ for the experiment) and predicted attenuation $(3 \%)$ is less than the measured value $(10 \%)$ but of the same order of magnitude. It 
explains why the apparent temperature near the edge in pulsed mode is different from the "bulk" temperature of the specimen.

To check that the coating layer is responsible for this artefact, a special specimen with an artificial variation of thickness is considered, all other material parameters being uniform (Fig. 9). The thin part has a thickness of about $50 \mu \mathrm{m}$, and the thick one around $100 \mu \mathrm{m}$, identical to a real specimen near the centre of the surface and near the edge. The model is used again to predict phase lag and attenuation for different loading frequencies and the measurements are performed under the same conditions. The comparisons presented in Fig. 10 show a good agreement between model and experimental results. Consequently, a simple way of preventing artefacts due to variations in coating thickness is to average a measured temperature field over several cycles.
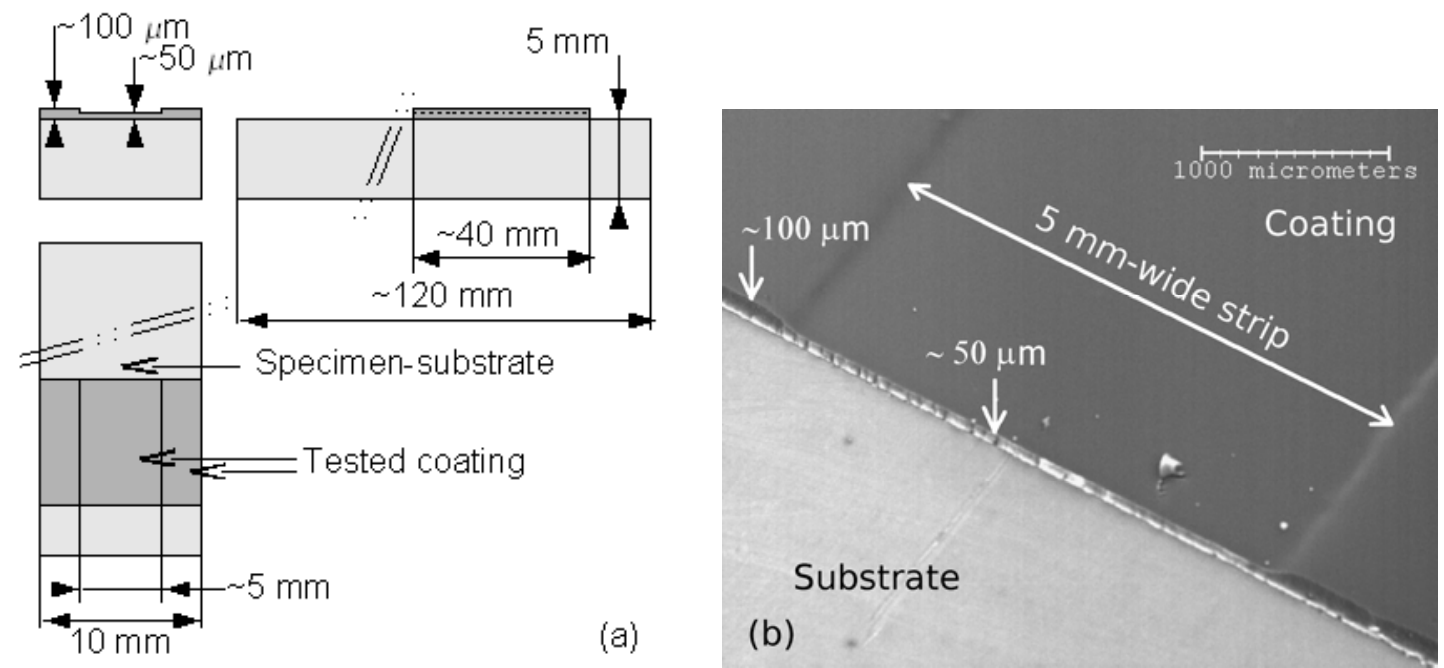

Figure 9: Substrate with an artificial variation of coating thickness: (a) geometry, (b) close-up view of the central $50 \mu \mathrm{m}$-thick strip transition.
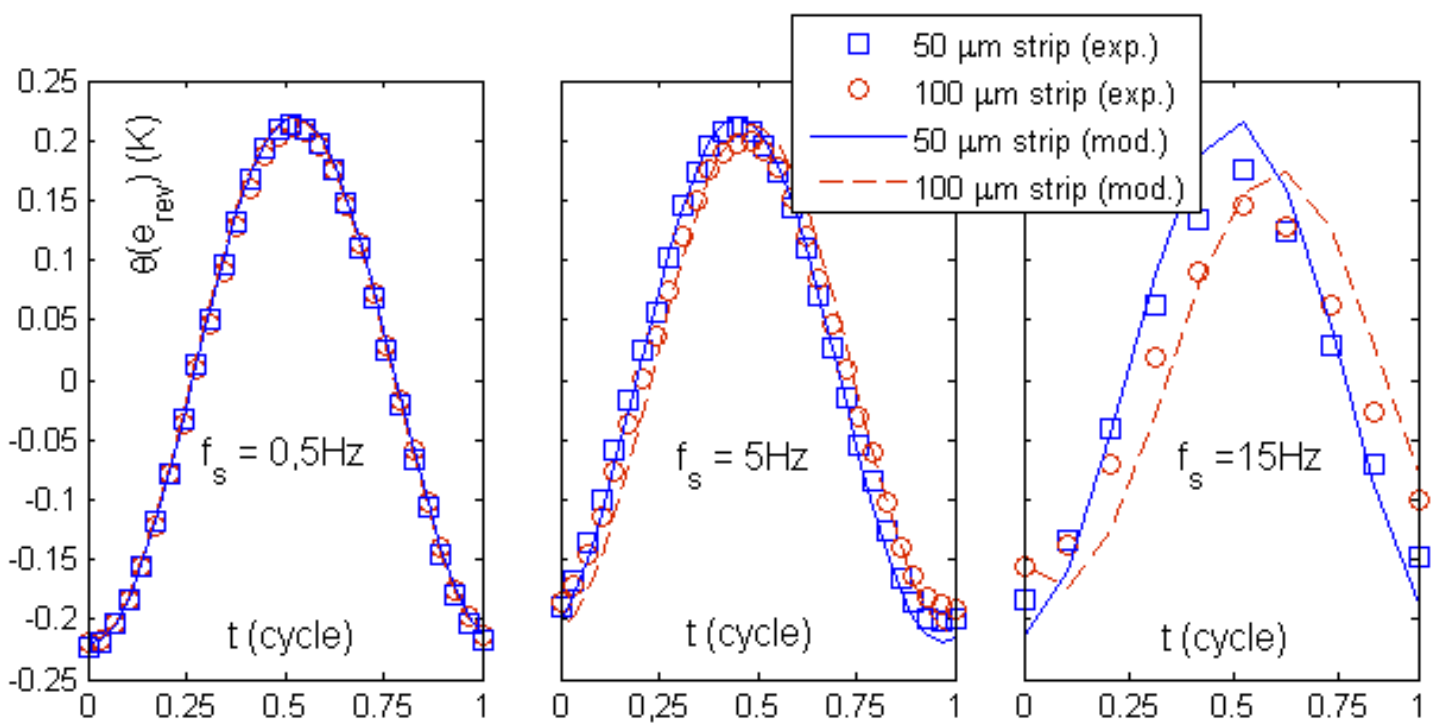

Figure 10: Measured temperature changes for the $50 \mu \mathrm{m}$ and $100 \mu \mathrm{m}$-thick strips and corresponding simulations for different loading frequencies.

\section{Convection noise}


Another artefact affecting especially edges of the specimen is the so-called "convection noise". Because of the very low magnitude of the sought temperature fluctuations, special care must be exercised for the thermal conditions. First, the "mean" influence of convection is removed because of the measurement principle itself, as long as the convection conditions are constant in time. Second, noise due to the turbulent component of convection has to be reduced. Last, the radiation of the surroundings has to be as constant as possible during the test.

The steadiness of convection conditions is ensured by a thick lined fabric, which prevents draughts to penetrate in the measurement area. The (type K) thermocouple being fixed at the end of the lower grip, it is checked that the thermal inertia of the grip is significantly greater than that of the specimen $(\approx 1 \mathrm{~h} \gg 30 \mathrm{~s})$. Consequently, the excess of heat coming from the loaded specimen and the actuator during a test does not change the temperature of the grips. Last, small drift of the air temperature is compensated because of the convection at the (actively cooled) surface of the black body placed just behind the specimen (Fig. 6).

The effect of convection turbulences on the instantaneous temperature is important, inducing high fluctuations, called "convection noise" on the edges of the specimen as shown in Fig. 11. Magnitudes over $\pm 45 \mathrm{mK}$ are measured during tests when the specimen undergoes the same environmental conditions as in a real test, but without mechanical loading. To lower this noise, the water-cooling ring put between the lower grip and the actuator is used. It allows one to decrease the differential temperature between the grips, monitored with thermocouples. When the lower grip is slightly colder than the upper one, the air turns to a static balance (i.e., temperature gradient to the top) contrary to a dynamic balance (i.e., gradient to the bottom). Moreover temperature magnitudes of the air fluctuations are lower. These two effects explain the difference of noise levels observed in Fig. 12.

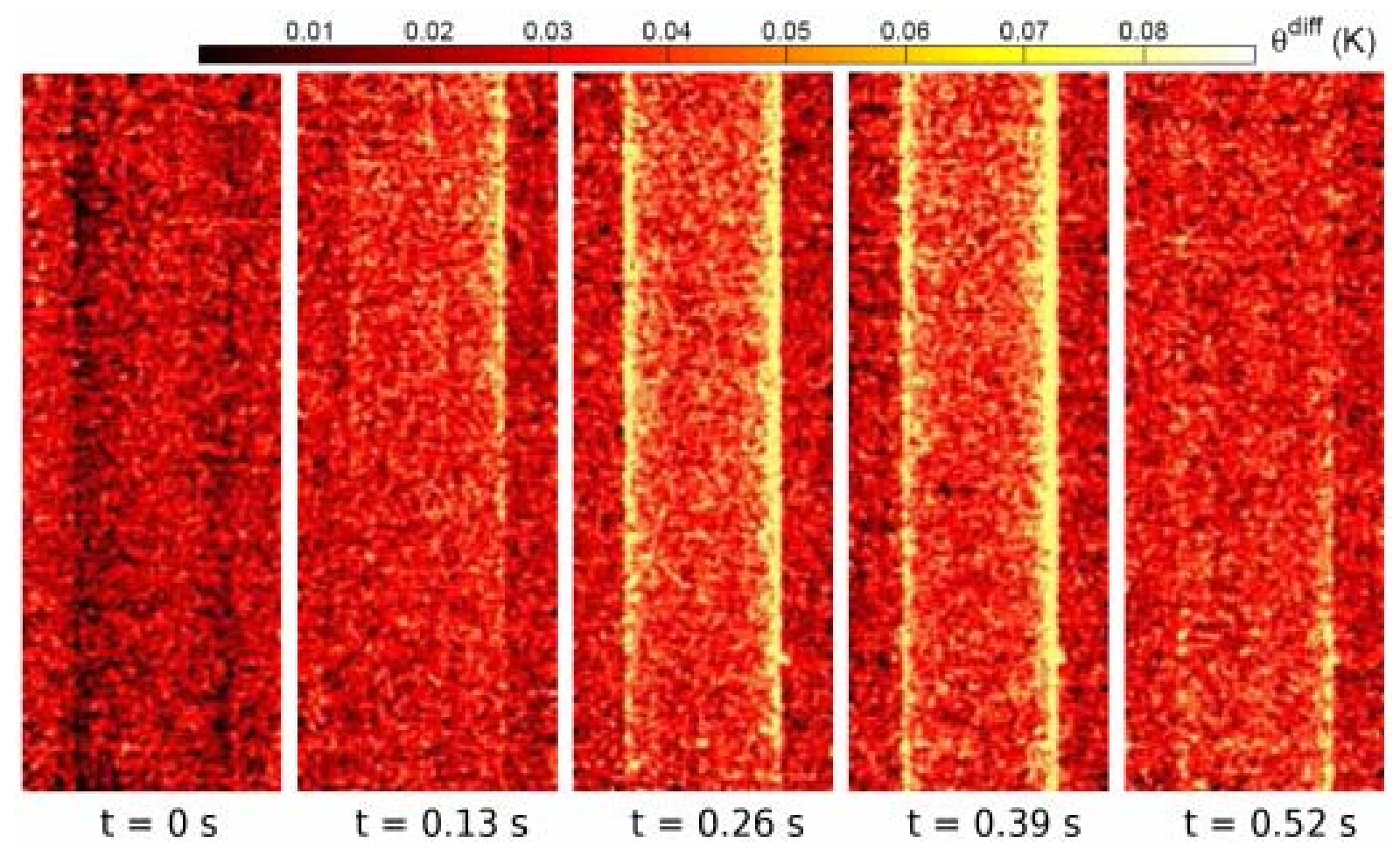


Figure 11: Excerpt of a movie shot with the specimen in the same environmental conditions as in a real test, but without cyclic loading. Temperature fluctuations are mainly observed on the edges due to air motion.

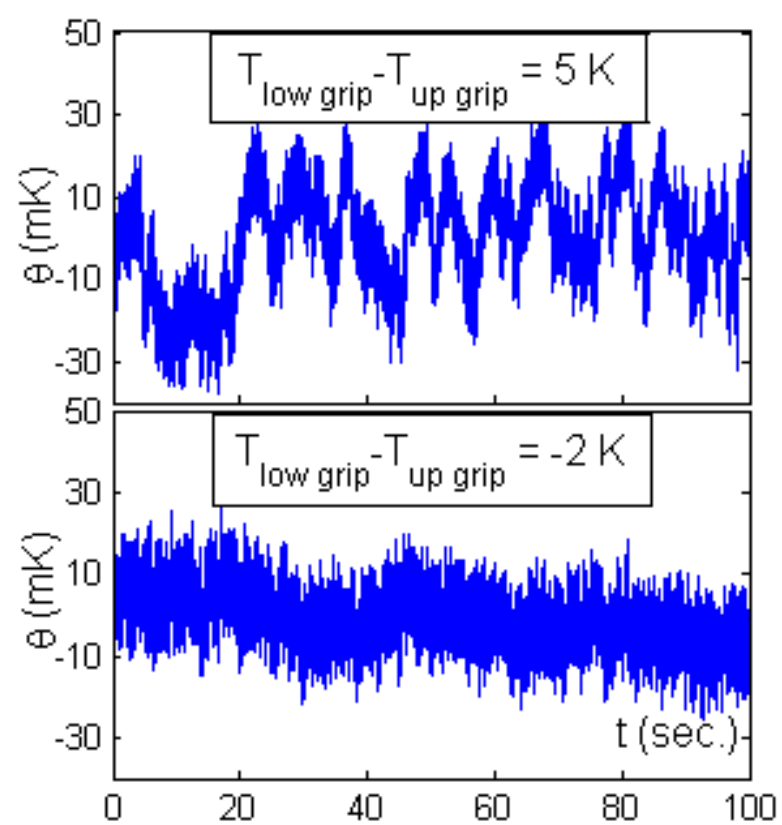

Figure 12: Fluctuations of the space-averaged temperature for uncooled and cooled lower grip.

Even though the effect of the grip temperature cooling on convection is important, fluctuations are still higher than the sought heterogeneities. It is thus proposed to homogenize the room temperature by mixing the air permanently by use of a fan placed in the measurement area (Fig. 6). A spectral analysis of the convection noise is shown in Fig. 13 (a). For natural convection, noise is roughly speaking proportional to $1 / f$ with a very high power spectral density at low frequencies, whereas for forced convection, noise tends to be closer to white noise with a power spectral density divided by 2 for low frequencies. As shown in Fig. 13 (b), for a time window longer than $0.6 \mathrm{~s}$, forced convection allows for the time average to be more efficient than with natural convection. The standard deviation of convection noise after filtering (time window $8 \mathrm{~s}$ ) is $1.1 \mathrm{mK}$ to be compared with $1.9 \mathrm{mK}$ for natural convection. Consequently, a combination of forced convection and long time averages is a viable solution to lower the influence of convection noise. 


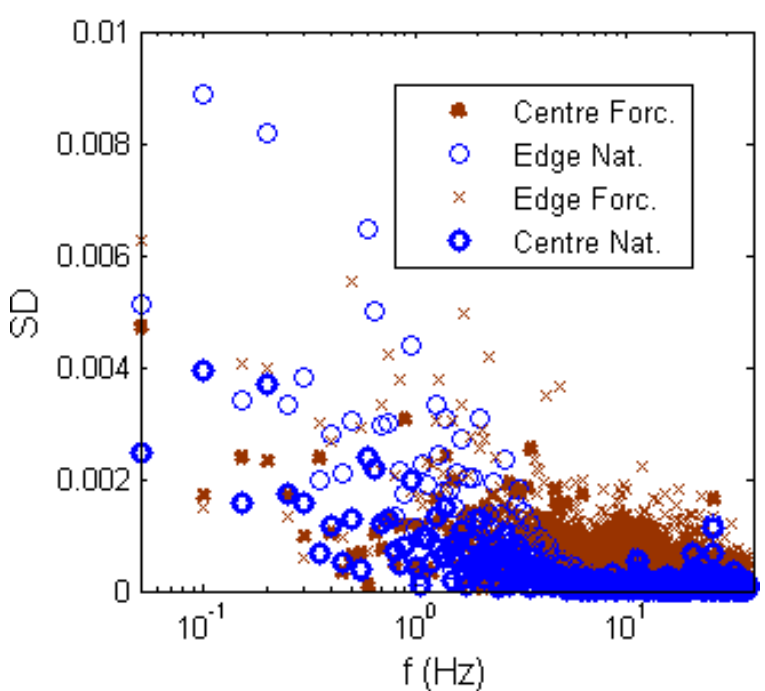

(a)

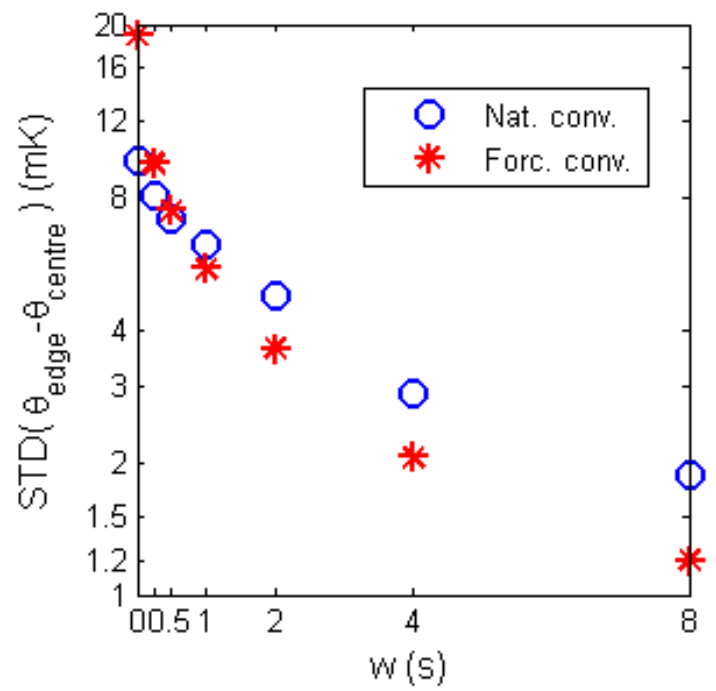

(b)

Figure 13: (a) Spectral analysis of temperature fluctuations in the centre and on the edge of a specimen for natural and forced convections. (b) Standard deviation of the fluctuations of the subtraction of centre and edge temperatures for different time-windows $w(s)$ and types of convection.

\section{Optical artefacts}

Last, optical artefacts must not affect measurements. Some of them are constant in time (Narcissus effect), some depend on the background temperature (background effect) and some on the temperature of the specimen (inverse Narcissus effect).

The usual "Narcissus effect" [27] is diminished by the use of the high emissivity coating. Because of the very short depth of field of the lens (i.e., less than $0.1 \mathrm{~mm}$ ), tilting the specimen in front of the camera is impossible without being out of focus. If the temperature of the detector is constant and the specimen still, reflection is constant in time, and therefore subject to cancellation by differential measurements. Unfortunately, during the test the very small motions of the lens previously described are unavoidable. From a theoretical point of view, a subtraction of the Narcissus effect is thus impossible, since the reflection is displaced in the same way as the physical points of the specimen during the DIC procedure. Because of the importance of rigid body motion corrections and the rather low gradient of the reflection due to the closeness of the lens, a solution is to apply this correction and neglect the slightest artefact due to the reflection displacement and later subtraction. Consequently this artefact is once more "deleted" by differential measurement.

Two other optical artefacts are due to the design of the microscopic lens itself, and its rather low transmission ( $85 \%)$. The first one, called "background effect", is explained by multiple reflections inside the lens, focusing the background radiations on the specimen. To illustrate this effect, the temperature of the specimen is measured in test conditions, except that there is no mechanical loading and the temperature of the black body (i.e., the background) is risen from $T_{C N 1}$ to $T_{C N 2}$ (around $40 \mathrm{~K}$ higher than $T_{C N 1}$ to ensure a favourable signal-to-noise ratio on the obtained images). Figure 14 shows the normalised difference between the two measurements. The magnitude of this effect is non-negligible, i.e., equivalent to $10 \%$ of the background variation. Therefore the use of the black body as stabilised background is necessary to allow for deletion by subtraction. 


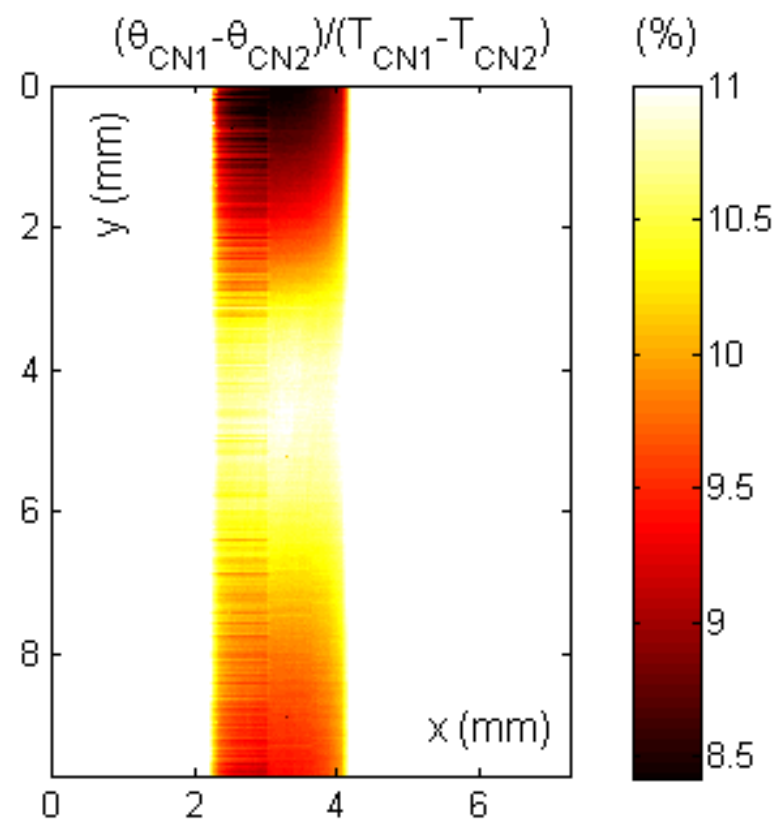

Figure 14: Normalized differential temperature between hot $\left(T_{C N 1}\right)$ and cold $\left(T_{C N 2}\right)$ backgrounds.

The last reported optical artefact is also based on multiple reflections. This time the source of the incoming rays is no longer the background but the specimen itself. Schematically, the route of the rays starts from the specimen, goes to the lens, then multiple reflections in the lens occur, and some reflected rays come back to the specimen, which partially reflects them a last time so that they re-enter the lens. This artefact will be thus referred to as "inverse Narcissus effect" because of its similarity with the Narcissus effect. This effect is both theoretically and experimentally studied.

A numerical model of the inverse Narcissus effect was obtained using a ray-tracing method (Zemax ${ }^{\circledR}$ software [28]). The G1 lens is completely modelled (i.e. lenses geometries and configuration, their bulk materials and optical surface properties, as well as the tubular body). A 1-mm in diameter uniform penny-shaped IR source is placed in the object plane. Figure 15 (a) shows the intensity of radiation coming back to the object plane. The white circle displayed on the right side of the image symbolises the position of the source. The inverse Narcissus effect creates a sharp "ghost image" of this source on the object plane, on the opposite side, with an intensity of around $1 \%$ of the intensity of the source.

Figure 15 (b) shows an experimental equivalent of the former simulation. For this purpose, a special target is designed, consisting of a steel diaphragm (aperture $1 \mathrm{~mm}$ ) with a mirror-polished surface. This target is put in front of the black body, with a chopper located between the black body and the target. Consequently, this complete set-up behaves as a uniform, non-directional, pulsed circular source. The low emissivity of the target surface ensures a high reflection of the rays that come back to the target after being reflected by the lens, so that they are easily detected. The pulsed source enables for a synchronous dataprocessing that improves the signal-to-noise ratio. Figure 15 (b) shows the rationalized differential radiation measured between the off and on state of the source. The white circle on the right side is this time the real source, whereas the halo on the opposite side is the "ghost image". Numerical and experimental results are in good agreement, namely, same magnitude ( $0.8 \%$ for experiment, $1.0 \%$ for simulation), same location (symmetry with respect to the optical axis) and same shape (identical to the source). Differences may come from the 
simulation (coefficient of reflection of each surface) and the experiment (flatness of the mirror-diaphragm, lens manufacturing defects).

Because of the use of a carbon coating on the specimens (contrary to the target), this artefact is even dimmer. Furthermore, the differential measurement leaves only the part of this artefact due to the difference of temperature between sequences 1 and 2, i.e., the sought heterogeneity. The remaining inverse Narcissus effect is thus assumed to be negligible with respect to the other ones listed above.

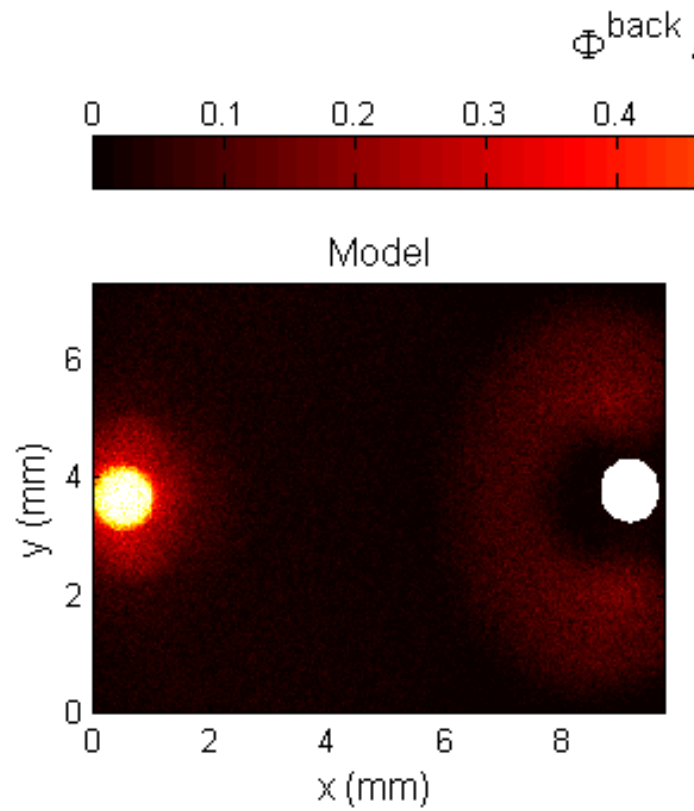

(a)

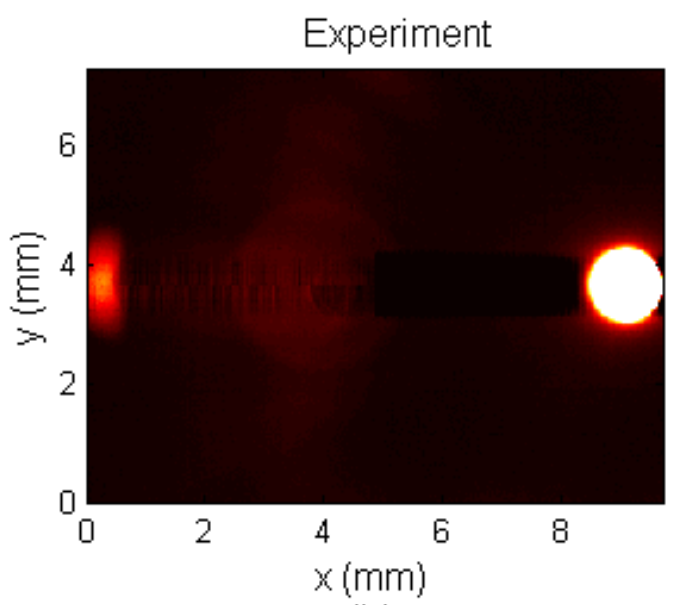

(b)

Figure 15: Inverse Narcissus effect. Numerical simulation and corresponding experimental measurement of the intensity of radiation coming back to the object plane in case of a uniform penny-shape source placed on the right (white circle)

\section{Results and discussion}

The complete protocol is now applied, i.e. all the different corrections / preventions of artefacts are now included, namely, most differential temperature method, asynchronous acquisition, coating, grip temperature control, turbulent convection control, rigid body motion correction, background temperature control.

22MnB5 specimens are first tested. Results are shown in Fig. 16(a). From a general point of view, one observes that temperature heterogeneities are, as expected, very different from those in Fig. 2 (d). With the "most differential measurement" method, the differential temperature no longer decreases near the edges of the specimen because of convection, but increases due to the sought surface effects, i.e. the partial decarburisation of the $50-\mu \mathrm{m}$ surface layer (Fig. 1 (a) \& (b)). It is important to note the respective magnitude of the different phenomena. The surface/bulk difference of temperature due to the "mean" convection is about $50 \mathrm{mK}$ (see Fig. 2 (d)). The magnitude of the convection noise is about $\pm 45 \mathrm{mK}$ (see Fig. 11), so that the method with several short cycles of stage I and stage II is necessary to lower this level (time averages are equivalent to $40 \mathrm{~s}$ for the present results). Eventually the measured surface / bulk difference of temperature is about $5 \mathrm{mK}$ (Fig. 16 (a)).

More precisely, one notices a dissymmetry of all the curves. However the same decarburised specimen (D) has been put in place, measured (D Face A), removed, turned respectively to the $y$-axis, put back in place and measured a second time (D Face B). These two results are slightly different but the dissymmetry has clearly not been flipped. This is 
evidence that the measurements are affected by a linear systematic error that is not related to material heterogeneities. A possible explanation of this dissymmetry is that of the forced convection set-up (use of a single fan on one side of the specimen). The effect of the "mean" convection is deleted by the differential measurement, but only its first order contribution. Considering a more realistic non-linear heat transfer model, the forced convection set-up may be the cause of this systematic error.

When studying surface effects, the small difference between curves (D Face A) and (D Face B) may be explained by a dissymmetry of material properties due to the manufacturing process. Despite this material defect, the measured magnitude of the surface / bulk difference of temperature (about $5 \mathrm{mK}$ ) is in good agreement with numerical estimations of temperature field during self-heating test [15], based on the average thermal behaviour of decarburized and non-decarburized steel sheets, and micrographic studies. Last, in the case of nondecarburized steel, surfaces are also hotter than the bulk (but to a lesser extent than in the previous case), which highlights another (dimmer) surface effect. These quantitative results, even though not allowing for calculations of heat sources, are deemed trustworthy.

The sandwiched specimens are also tested. Their interest is their assumed piece-wise constant behaviour (smooth temperature gradient, except small steps at the interfaces). The differential temperatures are shown in Fig. 16 (b) for two different loading amplitudes. For the sake of comparison, the two curves are shifted so that their temperatures in the central part are the same. The convection noise is lower than with $22 \mathrm{MnB} 5$ specimens, so that only 4-s time averages are necessary.

The observed dissymmetry is here due to a material dissymmetry (it was experimentally checked using the same method as before). The thickness of the thermal paste is not the same at each interface. The temperature steps at the interfaces of the steel sheets are clearly observable despite their magnitude (less than $2 \mathrm{mK}$, i.e. 10 times lower than the given NETD). The uncertainty, evaluated on the central sheet, is about $1 \mathrm{mK}$ while the time average is only $4 \mathrm{~s}$.

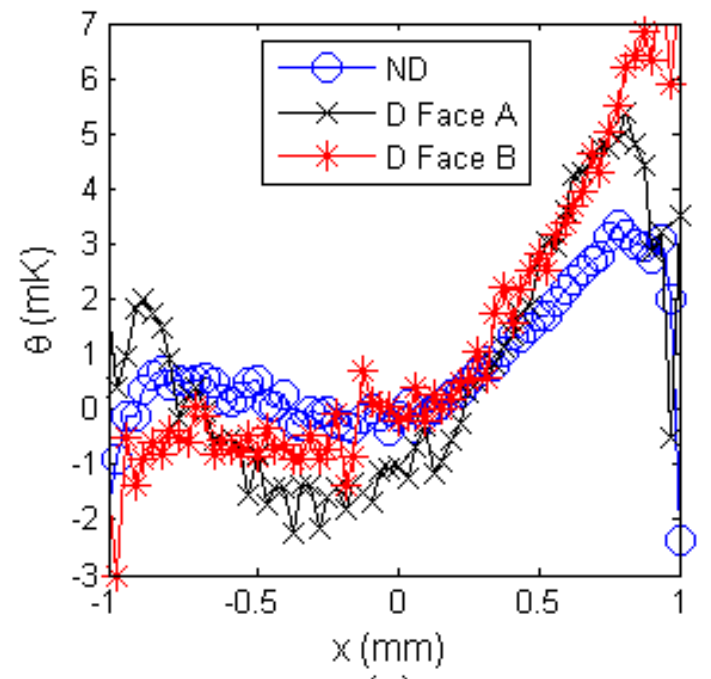

(a)

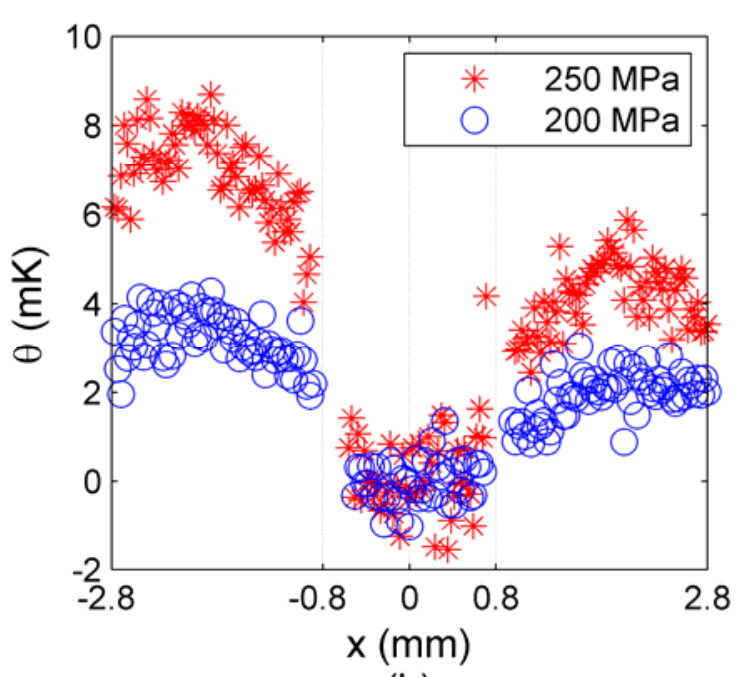

(b)

Figure 16: (a) Temperature field of both decarburised (D) and non-decarburised (ND) $22 \mathrm{MnB} 5$ steel sheets at $30 \mathrm{~Hz}$ (40 s time average). (b) Temperature field of the sandwiched specimen at $30 \mathrm{~Hz}$ (4 s time average). 


\section{Conclusion}

The aim of this study was to develop an experimental procedure allowing for microscopic infra-red measurements to detect low level heterogeneities (i.e., for temperature variations less than $10 \mathrm{mK}$ ) on thin metal sheets under cyclic loading. At the millimetre scale, the role of conductivity inside the specimen is so important that high level material heterogeneities lead to low level temperature heterogeneities. Several points are crucial:

- The convection influence is high at these levels of heterogeneities. To delete this effect, a "most differential measurement" is proposed, consisting in subtracting loaded and unloaded states of the specimen at the same mean temperature.

- The necessary high emissivity coating of the specimen introduces significant thermoelastic artefacts near geometric singularities (e.g., edges) upon cycling. Material heterogeneities may also create some. The use of asynchronous acquisition solves this problem.

- Rigid body motions of the specimen in the frame of the camera introduce nonnegligible errors during the subtraction of images. This effect is drastically reduced by a DIC based algorithm.

- In the present case, only the steady-state heterogeneity in the transverse $x$-direction of the sheet is sought, so that a time and space average is possible. Under these conditions, it is worth noting that the convection noise is more important after average (magnitude about $\pm 45 \mathrm{mK}$ ) than the measurement noise of the camera itself (about $\pm 1 \mathrm{mK}$ ). Sufficiently long time averages associated with the modification of turbulent convection condition reduce tremendously this noise.

- In the case of the used microscopic lens, one encounters complex, non-negligible, background effects. A steady-state temperature of the background is needed to avoid this spurious effect.

The complete experimental protocol allows one to achieve standard uncertainties as low as $1 \mathrm{mK}$. When applied to the case of partially decarburised steel sheets, the measured temperature field is clearly different for decarburised and non-decarburised steel sheets.

Last, such a type of measurements, although close to current technical limitations, is possible in a standard mechanical laboratory. This work is still in progress to explain the systematic error, to better understand the different optical artefacts, to reduce the global uncertainty level, and to apply the protocol to the study of other surface effects in high cycle fatigue.

\section{Acknowledgment}

The authors would like to thank Arcelor Research SA and Nippon Steel Corporation for their technical and financial support during this study, and Mr. Vaché (Cedip, France) for his helpful work on the simulation of the inverse Narcissus effect.

\section{References}

1 Galtier, A., Bouaziz O. and Lambert, A. (2002) Influence de la microstructure des aciers sur leurs propriétés mécaniques. Méc. Ind. 3, 457-462.

2 Doudard, C., Calloch, S., Hild, F., Cugy, P. and Galtier, A. (2005) A probabilistic two-scale model for high cycle fatigue life predictions. Fatigue Fract. Engrg. Mater. Struct. 28, 279288. 
3 Krapez, J.-C., Pacou, D. and Bertin, C. (1999) Application of lock-in thermography to rapid evaluation of fatigue limit in metals. Proceedings of the 5th AITA, Venezia (Italy).

4 La Rosa, G. and Risitano, A. (2000) Thermographic methodology for rapid determination of the fatigue limit of materials and mechanical components. Int. J. Fatigue 22, 65-73.

5 Boulanger, T., Chrysochoos, A., Mabru, C. and Galtier, A. (2004) Calorimetric analysis of dissipative and thermoelastic effects associated with the fatigue behavior of steels. Int. J. Fat. 26, 221-229.

6 Stromeyer, C.E. (1914) The determination of fatigue limits under alternating stress conditions. Proc. Roy. Soc. 90, 411-425.

7 Moore, H. F. and Kommers, J. B. (1921) Fatigue of metals under repeated stress. Chem. Met. Eng. 25, 1141-1144.

8 Harry, R., Joubert, F. and Gomaa, A. (1981) Measuring the actual endurance limit of one specimen using non-destructive method. J. Engrg. Mat. Techn. 103, 71-76.

9 Luong, M. P. (1992) Infrared thermography of fatigue in metals. SPIE 1682, 222-233.

10 Charkaluk, E. and Constantinescu, A. (2006) Estimation of the mesoscopic thermoplastic dissipation in High-Cycle Fatigue. C. R. Mécanique 334, 373-379.

11 Doudard, C., Calloch, S., Hild, F., Cugy, P. and Galtier, A. (2004) Identification of the scatter in high cycle fatigue from temperature measurements. C. R. Mécanique 332, 795-801.

12 Doudard, C., Poncelet, M., Calloch, S., Boué, C., Hild, F. and Galtier, A. (2007) Determination of an HCF criterion by thermal measurements under biaxial cyclic loading. Int. J. Fatigue 29 (4), 748-757.

13 Poncelet, M., Doudard, C., Calloch, S., Hild, F., Weber, B. and Galtier, A. (2007) Prediction of self-heating measurements under proportional and non-proportional multiaxial cyclic loadings. C. R. Mécanique. 335, 81-86.

14 Cazaud, R. (1959) La fatigue des métaux. Dunod, Paris (France).

15 Poncelet, M. (2007) Multiaxialité, hétérogénéités intrinsèques et structurales des essais d'auto-échauffement et de fatigue à grand nombre de cycles. PhD thesis, ENS Cachan .

16 Chrysochoos, A. and Louche, H. (2000) An infrared image processing to analyse the calorific effects accompanying strain localisation. Int. J. Eng Sci. 38, 1759-1788.

17 Honorat, V., Moreau, S., Muracciole, J.-M., Wattrisse, B. and Chrysochoos, A. (2005) Calorimetric analysis of polymer behaviour using a pixel calibration of an IRFPA camera. Int J. on Quantitative Infrared Thermography. V 2, 2, 153-172.

18 Darses, P. (2004) CEDIP Cameras User Manual.

19 Krapez, J.C., Pacou, D. and Gardette, G. (2000) Lock-in thermography and fatigue limit of metals. Proc. of QIRT'2000, Reims, 277-282.

20 Lemaitre, J. and Chaboche, J.L. (1990) Mechanics of Solid Materials. Cambridge University Press, Cambridge (UK).

21 Besnard, G., Hild, F. and Roux S. (2006) 'Finite-element' displacement fields analysis from digital images: Application to Portevin-Le Châtelier bands. Exp. Mech. 46, 789-803.

22 Sakagami, T., Nishimura, T., Yamaguchi, T., Kubo, N. (2006) Development of a new motion compensation technique in infrared stress measurement based on digital image correlation method. Nihon Kikai Gakkai Ronbunshu A / Trans Jpn Soc Mec Eng 72, 18531859.

23 Pottier, T., Moutrille, M.P., Le Cam, J.B., Balandraud, X., Grédiac, M. (2008) Study on the use of motion compensation techniques to determine heat sources. Application to large deformations on cracked rubber specimens. Exp. Mech. In Press.

24 Gaussorgues, G. (1980) La thermographie infrarouge. Technique et documentation, 1ère édition.

25 Pajani, D. (2001) Thermographie : principes et mesure. Technique de l'ingénieur, page R2740. 
26 McKelvie, J. (1987) Consideration of the surface temperature response to cyclic thermoelastic heat generation. SPIE Stress Analysis by Thermoelastic Techniques 731, 44-53.

27 Gross, H., Zügge, H., Blechinger, F. and Achtner, B. (2008) Handbook of Optical Systems Survey of Optical Instruments. Wiley-VCH, Berlin.

28 ZEMAX: Software For Optical System Design, http://www.zemax.com/ 